\title{
Zero estimates with moving targets
}

W. D. Brownawell and D. W. Masser

Departement Mathematik und Informatik

Preprint No. 2016-25

November 2016

Universität Basel

CH-4051 Basel

www.math.unibas.ch 


\title{
Zero estimates with moving targets
}

W.D. Brownawell and D.W. Masser

\begin{abstract}
Zero estimates have a classical history in diophantine approximation and transcendence, as well as more recent applications to counting rational points on analytic sets. We give some examples showing that the sharpest conceivable results can be false, and in some cases the natural guess has even to be doubled. A by-product is a "non-degenerate" case of the (unproved) "Zilber Nullstellensatz" in connexion with "Strong Exponential Closure".
\end{abstract}

2010 MSC codes. 03C99, 30C15, 32A60, 33E05.

1. Introduction. Let $f=f(z), g=g(z)$ be entire or meromorphic functions of a single complex variable $z$. If they are analytic at some $z=a$, then the simplest argument from linear algebra enables us to find for each pair of integers $L \geq 0, M \geq 0$ a non-zero polynomial $P=P(X, Y)$ in $\mathbf{C}[X, Y]$, of degree at most $L$ in $X$ and of degree at most $M$ in $Y$, such that the function $\phi=\phi(z)=P(f, g)$ vanishes at $z=a$ to order at least

$$
T=(L+1)(M+1)-1 .
$$

For this means $\phi^{(t)}(a)=0$ with the derivatives $\phi^{(t)}=(\mathrm{d} / \mathrm{d} z)^{t} \phi$ for $t=0,1, \ldots, T-1$, which are $T$ homogeneous linear equations in the $T+1$ unknown coefficients of $P$.

In applications to the theory of diophantine approximation and transcendence, a key role is played by upper bounds for such orders of vanishing. It usually suffices to have a bound of the form $C(L+1)(M+1)$, where $C$ is independent of $L, M$. The argument above shows $C<1$ is not possible; but there are rather few $f, g$ for which a result is known even with $C<2$. It is easy to check for $f=z, g=e^{z}$, and even that the order cannot exceed $T$. But if $\wp$ denotes a Weierstrass elliptic function, then despite much work of many authors, it remains unknown for $f=z, g=\wp(z)$. To our knowledge the best result is due to Gelfond in Lemma 4 (page 251) of [F], which implies an upper bound $2 T+\frac{1}{2}(L+1)^{2}$.

The theme of the present paper is that the arguments from linear algebra can sometimes be misleading, and we illustrate this with two classes of examples. 
First we shall prove that the order of vanishing of $P(z, \wp(z))$ can be at least $T+1$ if $a$ is carefully chosen.

Theorem 1. Given $\wp$ and positive integers $L, M$, there is a point a at which $\wp$ is analytic, and a non-zero polynomial $P(X, Y)$ of degree at most $L$ in $X$ and of degree at most $M$ in $Y$, such that $P(z, \wp(z))$ has a zero of order at least $L M+L+M+1$ at $z=a$.

As mentioned above, this is false for the exponential analogue $P\left(z, e^{z}\right)$.

We believe that the order of vanishing of $P(z, \wp(z))$ can even be at least $T+2$ if $\wp$ is carefully chosen as well as $a$. That is, we have

Conjecture. Given positive integers $L, M$, there is $\wp$ and a point a at which $\wp$ is analytic, and a non-zero polynomial $P(X, Y)$ of degree at most $L$ in $X$ and of degree at most $M$ in $Y$, such that $P(z, \wp(z))$ has a zero of order at least $L M+L+M+2$ at $z=a$.

This is even more false, and even somewhat meaningless, for the exponential analogue. We give some evidence for small $L, M$ to support the conjecture.

Thus instead of treating $a$ and $\wp$ as fixed, we are thinking of them as moving targets.

Here moving the targets has a relatively modest effect. We can exhibit a much greater effect by considering several zeroes instead of a single one. Thus given $a_{1}, \ldots, a_{N}$ with $N \leq T$ where $f, g$ are analytic, the linear algebra arguments show that we can find $P \neq 0$, again of degree at most $L$ in $X$ and of degree at most $M$ in $Y$, with $\phi=P(f, g)$ having zeroes at these points. Choosing $N=T$, we get $T$ different zeroes. Now here the effect of moving the targets $a_{1}, \ldots, a_{N}$ is enormous: a given $\phi$, such as $e^{z}-1$ or even $e^{z}-z$, will usually have infinitely many zeroes, and in this case we can improve the number of zeroes from $T$ to $\infty$.

The situation changes if we consider repeated zeroes; that is, with multiplicity at least two.

As $\left(e^{z}-1\right)^{\prime}=e^{z} \neq 0$, the function $e^{z}-1$ has no repeated zeroes. Similarly $\left(e^{z}-z\right)^{\prime}=$ $e^{z}-1=z-1 \neq 0$ if $e^{z}=z$, so the same is true of $e^{z}-z$.

Of course we can construct repeated zeroes by linear algebra. Given $a_{1}, \ldots, a_{N}$ now with $2 N \leq T$, we can find $P \neq 0$ such that $\phi$ has repeated zeroes at these points. By choosing $N$ as the integer part $\left[\frac{T}{2}\right]$ we get at least $\left[\frac{T}{2}\right]$ such zeroes (without multiplicity). We believe that this can always be essentially doubled. However if $P$ happens to be a 
square $P_{0}^{2}$ here, then we can usually get infinitely many repeated zeroes just by considering $\phi_{0}=P_{0}(f, g)$. Thus in order to exclude such trivialities we should search among squarefree $P$. In fact we are able to find $P$ which are even irreducible over $\mathbf{C}$.

We continue our theme by proving that if $M=1$ then indeed $\phi=P\left(z, e^{z}\right)$ can have as many as $T-1=2 L$ such zeroes if the targets $a_{1}, \ldots, a_{N}$ are carefully chosen.

Theorem 2. Given a positive integer $L$, there is a non-zero irreducible polynomial $P(X, Y)$ of degree at most $L$ in $X$ and of degree at most 1 in $Y$, such that $P\left(z, e^{z}\right)$ has at least $2 L$ different zeroes of order at least 2.

A relatively simple argument (see section 6 ) shows also that the expected $T=2 L+1$ is not possible. But for general $M$, even here for the simple exponential function, the correct upper bounds seem to be unknown. One can show following [BM] (see just below) that if $P$ is squarefree, then $P\left(z, e^{z}\right)$ has at most finitely many repeated zeroes and in fact at most $2 L M$. For general $M$ we do not know if this can be halved.

Actually in applications one often needs upper bounds for sums of orders of zeroes at various points (usually with some sort of group structure corresponding to a group variety - see Philippon's paper $[\mathrm{P}]$ for some very general results). Thus the linear algebra arguments show that we can find $P \neq 0$ with

$$
\sum_{n=1}^{N} \operatorname{ord}\left(\phi ; a_{n}\right) \geq T
$$

where $\operatorname{ord}(\phi ; a)$ is the order of vanishing of $\phi=P(f, g)$ at $a$. Incidentally here as well the correct upper bounds for exponential functions are unknown when $N \geq 2$. Of course the bound $N T$ is trivial. This for $L=M=1$ is $3 N$. In turn this for $N=2$ is 6 ; and when $\phi=P\left(z, e^{z}\right)$ it is not too hard to improve this to 4 when $a_{1} \neq a_{2}$, which is then best possible. But what happens for $N=3$ here?

Baker and Wüstholz in [BW] (pages 102,103) have also commented on the problems of proving the zero estimates expected from linear algebra:

We remark that the most troublesome factor here is $r$ ! that appears on the right-hand side of ( ) and arises as a consequence of the multihomogeneous degree theory; we have no heuristic explanation as to why this factor should occur and indeed it would seem that it should not be present if one counts conditions as usual in transcendence theory and one believes in the philosophy of Dyson type lemmas. The elucidation of this phenomenon would be of much interest. 
And Wüstholz earlier in [W] (page 193) had speculated: It seems to have something to do with infinity.

We certainly have no explanation either. The simplest non-trivial case of the arguments in [BW], [W] are just with our $\phi=P\left(z, e^{z}\right)$. If $\phi$ has a large order zero then so has the derivative $\phi^{\prime}$. This is also $P^{\#}\left(z, e^{z}\right)$ for $P^{\#}$ in $\mathbf{C}[X, Y]$ (here $P^{\#}=\Delta P$ for the differential operator $\Delta=(\partial / \partial X)+Y(\partial / \partial Y)$, not needed here). We may usually form the resultant $Q$ of $P, P^{\#}$ with respect to $Y$, a polynomial combination of $P, P^{\#}$. So $Q(z)$ is a corresponding combination of $\phi, \phi^{\prime}$ and also has a large order zero. But the zeroes of $Q(z)$ are easy to count in terms of its degree. This is of course the basic simple idea in [BM]. But the nasty surprise is that the natural bound for the degree, $2 L M$ in our situation, is roughly our quantity $T=L M+L+M$ multiplied by another troublesome factor $2=2$ !. Thus it is difficult to prevent this factor entering the zero estimates.

An example is that above for repeated zeroes. Each of these gives a zero of $Q$. One can check that $Q$ is not identically zero (again see section 6 for the case $M=1$ ). So there are at most $2 L M$ of them as claimed.

It is amusing to note that Theorem 2 says that in the case $M=1$ this bound is sharp, so we seem to have shown that this particular troublesome factor is actually needed; and furthermore by using resultants!

Our concept of moving targets is not quite the one more usually encountered in Vojta Theory. But it may have some relevance to Bombieri-Pila-Wilkie Theory, where refinements of the proofs call for zero estimates involving unknown points certainly without any group structure (see for example Proposition 1 of [Mas] page 2038). However there we have no multiplicities.

The paper is arranged as follows. In section 2 we prove Theorem 1 using Wronskians and degenerate elliptic functions. Then in section 3 we discuss some evidence for the Conjecture.

In section 4 we prove an auxiliary result (Proposition 1) about polynomials which arise from the resultant used above. Then section 5 contains another auxiliary result (Proposition 2) about exponential-polynomial equations. This is relevant to Zilber's "Strong Exponential Closure Axiom" for "pseudoexponential fields", which over $\mathbf{C}$ becomes a conjecture, here proved in a significant special case. Finally it is a relatively simple matter in section 6 to prove Theorem 2 .

As this paper was being completed, we received a copy of the preprint [DFT], in which 
D'Aquino, Fornasiero and Terzo also prove our Proposition 2 along similar lines (based on circulated notes of the present second author).

2. Proof of Theorem 1. We start off with an easy

Lemma 1. Let $f_{1}, \ldots, f_{S}$ be meromorphic functions with Wronskian $W$.

(i) If $f_{1}, \ldots, f_{S}$ are analytic at a point a with $W(a)=0$, then there are constants $c_{1}, \ldots, c_{S}$, not all zero, such that $c_{1} f_{1}+\cdots+c_{S} f_{S}$ has a zero of order at least $S$ at $a$.

(ii) If $f_{1}, \ldots, f_{S}$ have poles of orders $1, \ldots, S$ respectively at a point $b$, then $W$ has a pole of order $S^{2}$ at $b$.

Proof. For (i) again the natural order is $S-1$, so there is here too an extra 1 . The result is practically obvious: the Wronskian matrix with entries $f_{s}^{(t-1)}(a)(s, t=1, \ldots, S)$ is singular and so the columns are linearly dependent.

For (ii) we can argue by induction on $S$ using the well-known fact that the Wronskian of $g f_{1}, \ldots, g f_{S}$ is $g^{S} W$. With $g=1 / f_{1}$ we reduce to a Wronskian of $S-1$ functions, which after multiplication also by $1 / f_{1}$ have poles of orders $1, \ldots, S-1$. The result follows because $S^{2}=S+(S-1)+(S-1)^{2}$.

Thus to prove Theorem 1 we take $S=(L+1)(M+1)$ and the $f_{s}$ as the $z^{l} \wp^{m}(l=$ $0, \ldots, L, m=0, \ldots, M)$. Now the Wronskian $W$ is actually an elliptic function; that is, no powers of $z$ survive the determinant expansion. This can be seen for example by noting that if $\omega$ is any period of $\wp$ then replacing $z$ by $z+\omega$ has the effect of acting on the $f_{s}$ by a unimodular matrix. This action extends to the whole Wronskian matrix. So by taking determinants we see that $W(z)=W(z+\omega)$. Thus $W$ is indeed elliptic. It is certainly non-zero, because $z, \wp$ are well-known to be algebraically independent and so the $f_{s}$ are linearly independent. So as long as we can show that $W$ is not identically constant, it has a zero at some $z=a$ and we are done thanks to Lemma 1(i).

If $L=1$ we can show this as follows. After rearrangement the $z^{l} \wp^{m}(l=0, \ldots, L, m=$ $0, \ldots, M)$ have poles of orders $-1,0,1, \ldots, 2 M$ at $z=0$. Multiplying by $1 / z^{2}$, we find from Lemma 1(ii) that $W$ has a pole at $z=0$ of order $-2 S+(2 M+2)^{2}=S(S-2) \geq 8$, and indeed cannot be constant.

So henceforth we assume that $L \geq 2$. Now from the famous differential equation $\wp^{\prime 2}=4 \wp^{3}-g_{2} \wp-g_{3}$ we see that $W$ is a polynomial in $\wp, \wp^{\prime}, g_{2}, g_{3}$. If we give these the usual weights 2,3,4,6 respectively, then the polynomial is quadrihomogeneous. Thus 
if $W$ were identically constant, then it would be a (non-zero weighted) polynomial in the invariants $g_{2}, g_{3}$. So it is a product of factors $\alpha g_{2}^{3}-\beta g_{3}^{2}$. If some projective $(\alpha, \beta)$ is not $(1,27)$, then we could make the factor vanish by specializing to a particular $\wp$. But this would mean that the particular $z, \wp$ are algebraically dependent. The only way out is that $W$ has the form $\gamma\left(g_{2}^{3}-27 g_{3}^{2}\right)^{k}$ with $k \geq 0$.

If $k \neq 0$ then we can make this vanish by specializing to a degenerate $\wp$. A suitable "multiplicative" one is

$$
\wp_{\infty}(z)=-\frac{1}{3}+\sin ^{-2}(z)
$$

which satisfies the differential equation

$$
\wp_{\infty}^{\prime 2}=4 \wp_{\infty}^{3}-\frac{4}{3} \wp_{\infty}-\frac{8}{27}=4\left(\wp_{\infty}-\frac{2}{3}\right)\left(\wp_{\infty}+\frac{1}{3}\right)^{2}
$$

with

$$
g_{2}=\frac{4}{3}, g_{3}=\frac{8}{27}, \quad g_{2}^{3}-27 g_{3}^{2}=0 .
$$

Now we could deduce that $z, \wp_{\infty}$ would be algebraically dependent, still impossible.

If $k=0$ we could consider the "additive" degenerate $\wp_{\infty}(z)=1 / z^{2}$ satisfying $\wp_{\infty \infty}^{\prime 2}=4 \wp_{\infty \infty}^{3}$ with $g_{2}=g_{3}=0$. Then $W=\gamma$ stays non-zero. Here $z, \wp_{\infty \infty}$ do become algebraically dependent; and, more to the point, because $L \geq 2$ the $z^{l} \wp_{\infty \infty}^{m}(l=$ $0, \ldots, L, m=0, \ldots, M)$ become linearly dependent (for example just $1=z^{2} \wp_{\infty}$ ), another contradiction.

This completes the proof of Theorem 1. The use of various degenerate elliptic functions suggests that generalizations to abelian functions may not be so easy, possibly involving dimensions of various moduli spaces.

Here is what the proof gives when $L=M=1$. For any $x=\wp(a)$ satisfying

$$
x^{4}+\frac{1088391168 g_{2}^{3} g_{3}^{2}}{\left(g_{2}^{2}-27 g_{3}^{2}\right)^{2}} x^{2}+\frac{203119913336832 g_{2}^{3} g_{3}^{4}}{\left(g_{2}^{2}-27 g_{3}^{2}\right)^{3}} x+\frac{296148833645101056 g_{2}^{6} g_{3}^{4}}{\left(g_{2}^{2}-27 g_{3}^{2}\right)^{4}}=0
$$

(exhibiting the degeneracy in action) there is a non-zero function

$$
p_{00}+p_{10} z+p_{01 \wp(z)+p_{11} z \wp(z)}
$$

having a zero of order at least 4 at $z=a$.

3. The Conjecture. One can find some support for the Conjecture by pushing Lemma $1(\mathrm{i})$ a bit further to involve $W(a)=W^{\prime}(a)=0$. 
Thus for $L=1, M=1$ one finds with $\wp^{\prime 2}=4 \wp^{3}-4 \wp($ so $j=1728)$ and $a$ with $\wp(a)=i, \wp^{\prime}(a)=2-2 i$ (corresponding to a point of order 4 ) that (2.1) with

$$
p_{00}=-2 i a-i-1, p_{10}=2 i, p_{01}=-2 a+1-i, p_{11}=2
$$

has a zero of order 5 at $z=a$; here $T=3$. In fact it is the only example up to elliptic isomorphism, Galois conjugation, sign change and scalar multiplication.

And for $L=2, M=1$ one finds with $\wp^{\prime 2}=4 \wp^{3}-4$ (so $j=0$ ) and $a$ with $\wp(a)=$ $1, \wp^{\prime}(a)=0$ (corresponding to a point of order 2 ) that the function

$$
p_{00}+p_{10} z+p_{20} z^{2}+p_{01 \wp} \wp(z)+p_{11} z \wp(z)+p_{21} z^{2} \wp(z)
$$

with

$$
p_{00}=1+2 a^{2}, p_{10}=-4 a, p_{20}=2, p_{01}=-1+a^{2}, p_{11}=-2 a, p_{21}=1
$$

has a zero of at least order 7 (actually order 8 ) at $z=a$; here $T=5$. There are similar examples with $\wp(a)$ any primitive cube root of unity and again $\wp^{\prime}(a)=0$.

And also for $\wp^{\prime 2}=4 \wp^{3}-10 \wp-6\left(\right.$ so $\left.j=\frac{432000}{7}\right)$ and for example

$$
\wp(a)=-1+\frac{\sqrt{2}}{2}, \quad \wp^{\prime}(a)=i \sqrt{6-2 \sqrt{2}}
$$

(also corresponding to a point of order 4).

And for $L=3, M=1$ one finds again $\wp^{\prime 2}=4 \wp^{3}-4($ so $j=0)$ and $a$ with $\wp(a)^{3}=1$, as well as $\wp^{\prime 2}=4 \wp^{3}-50 \wp+70$ (so $j=-\frac{2160000}{73}$ ) and $a$ such that $x=\wp(a)$ satisfies $x^{4}-4 x^{3}+25 x^{2}-90 x+\frac{345}{4}=0$ (apparently not torsion) giving a zero of order at least 9 at $z=a$; here $T=7$. In fact there are examples with the other $j$-values

$$
j=\frac{46008000 \pm 7560000 \sqrt{37}}{11} .
$$

Going up to $L=4, M=1$ one finds six more $j$-values giving a zero of order at least 11; namely

$$
j=\frac{864000}{59}, j=\frac{-101628000 \pm 15876000 \sqrt{41}}{61} .
$$

and the solutions of

$$
551 j^{3}-690888528000 j^{2}+10273651200000000 j+20155392000000000000=0 .
$$


And finally for $L=2, M=2$ one finds (as well as $j=1728$ )

$$
j=-\frac{54000}{79}, \quad j=\frac{205875000 \pm 25515000 \sqrt{65}}{47}
$$

and the solutions of

$$
233 j^{3}-23878800 j^{2}+2208870000000 j+1968300000000000=0 .
$$

In fact as $L, M$ increase it seems very likely that the number of $j$-values increases; and that is why we believe our conjecture.

4. Preliminaries. A key result, actually one of algebraic independence (see section 6), for dealing with the case $M=1$ in Theorem 2 is the following, where we identify non-zero polynomials of degree at most $D$ up to constant multiples with points of projective $\mathbf{P}_{D}$.

Proposition 1. The formula

$$
Q=P_{0} P_{1}+P_{0} P_{1}^{\prime}-P_{0}^{\prime} P_{1}
$$

defines a surjective morphism from $\mathbf{P}_{L} \times \mathbf{P}_{L}$ to $\mathbf{P}_{2 L}$.

Proof. It is a morphism because if $P_{0} \neq 0$ has exact degree $l_{0} \geq 0$ and $P_{1} \neq 0$ has exact degree $l_{1} \geq 0$ then $Q$ has exact degree $l_{0}+l_{1} \geq 0$ so $Q \neq 0$.

We assume it is not surjective and we will obtain a contradiction.

Thanks to projectivity the image is a proper subvariety of $\mathbf{P}_{2 L}$ (see for example [M] page 33). In fact the morphism defines a map from $\mathbf{C}^{L+1} \times \mathbf{C}^{L+1}$ to $\mathbf{C}^{2 L+1}$ and the image of this then lies in a proper subvariety. We introduce coordinates by writing

$$
P_{0}=\sum_{l=0}^{L} p_{l 0} X^{l}, P_{1}=\sum_{l=0}^{L} p_{l 1} X^{l}, Q=\sum_{k=0}^{2 L} q_{k} X^{k},
$$

so that the $q_{k}$ are polynomials in the $p_{l m}$. Then the Jacobian $J$ with $2 L+2$ rows

$$
\left(\frac{\partial q_{0}}{\partial p_{l m}}, \ldots, \frac{\partial q_{2 L}}{\partial p_{l m}}\right) \quad(l=0,1, \ldots, L ; m=0,1)
$$

acquires in the usual way a non-trivial relation between the $2 L+1$ columns, and therefore has rank at most $2 L$. 
This in turn delivers two linearly independent row relations. Thus for generic $P_{0}, P_{1}$ in $\mathbf{C}[X]$ (of degrees at most $L$ so exactly $L$ ) there are two linearly independent row relations in the corresponding $J$.

We now introduce new polynomials to express a given row relation

$$
\sum_{l=0}^{L}\left(a_{l 0} \frac{\partial q_{k}}{\partial p_{l 0}}+a_{l 1} \frac{\partial q_{k}}{\partial p_{l 1}}\right)=0 \quad(k=0,1, \ldots, 2 L) .
$$

It is equivalent to the single relation

$$
\sum_{l=0}^{L}\left(a_{l 0} Q_{l 0}+a_{l 1} Q_{l 1}\right)=0
$$

where

$$
\begin{aligned}
& Q_{l 0}=\frac{\partial Q}{\partial p_{l 0}}=X^{l} P_{1}+X^{l} P_{1}^{\prime}-l X^{l-1} P_{1} \quad(l=0,1, \ldots, L), \\
& Q_{l 1}=\frac{\partial Q}{\partial p_{l 1}}=X^{l} P_{0}-X^{l} P_{0}^{\prime}+l X^{l-1} P_{0} \quad(l=0,1, \ldots, L) .
\end{aligned}
$$

Then (4.1) can be written

$$
A_{0} P_{1}+A_{0} P_{1}^{\prime}-A_{0}^{\prime} P_{1}+A_{1} P_{0}-A_{1} P_{0}^{\prime}+A_{1}^{\prime} P_{0}=0
$$

with

$$
A_{0}=\sum_{l=0}^{L} a_{l 0} X^{l}, \quad A_{1}=\sum_{l=0}^{L} a_{l 1} X^{l} .
$$

Now (4.2) is satisfied with

$$
A_{0}=P_{0}, \quad A_{1}=-P_{1}
$$

and so we recover one of the two row relations mentioned above.

However there is a second such relation independent of this. So by taking linear combinations we could assume that there is a relation with $A_{0}$ of degree at most $L-1$. Now (4.2) shows that also $A_{1}$ has degree at most $L-1$. Then (4.2) gives a set of $2 L$ homogeneous linear equations in the $2 L$ surviving coefficients of $A_{0}, A_{1}$, and we will show that the (generic) determinant does not vanish, giving the contradiction $A_{0}=A_{1}=0$.

Actually it suffices to show that for some specialized $P_{0}, P_{1}$ the system (4.2) implies $A_{0}=A_{1}=0$; for then the specialized determinant does not vanish. Computational experiment guided the unlikely-looking choice

$$
P_{0}=X^{L}, \quad P_{1}=X^{L}+t
$$


with any irrational $t$. Now (4.2) can be rearranged to

$$
X^{L-1}\left(X A_{0}+L A_{0}-X A_{0}^{\prime}+X A_{1}-L A_{1}+X A_{1}^{\prime}\right)+t\left(A_{0}-A_{0}^{\prime}\right)=0
$$

Thus $X^{L-1}$ divides $A_{0}-A_{0}^{\prime}$. By comparing degrees we see that either $A_{0}=A_{0}^{\prime}$ or (without loss of generality) $X^{L-1}=A_{0}-A_{0}^{\prime}$.

The first possibility clearly implies $A_{0}=0$; but then (4.3) gives $X A_{1}-L A_{1}+X A_{1}^{\prime}=0$ which implies $A_{1}=0$ also by comparing degrees. So in the first case $A_{0}=A_{1}=0$ as desired.

The second possibility $X^{L-1}=A_{0}-A_{0}^{\prime}$ determines $A_{0}$ uniquely in $\mathbf{Q}[X]$. Now (4.3) reads (after dividing by $X^{L-1}$ )

$$
X^{L}+L A_{0}+X A_{1}-L A_{1}+X A_{1}^{\prime}+t=0 .
$$

By looking at the coefficients of $X^{L}, X^{L-1}, \ldots, X$ successively it can be seen that

$$
a_{L-1,1}, a_{L-2,1}, \ldots, a_{01}
$$

are in $\mathbf{Q}$. However the constant terms then show that $-L a_{01}+t$ is also in $\mathbf{Q}$. This means that the second possibility cannot occur, and thereby completes the proof of Proposition 1.

5. Exponential-algebraic equations. Here we state and prove our result on the existence of solutions.

In one variable it is well-known and easy to prove that if $P(X, Y)$ is any polynomial involving $X$ and $Y$ then $P\left(z, e^{z}\right)$ has a complex zero. For example we can solve the equation $e^{z}=F(z)$ with a non-constant polynomial $F$. And if $F$ is allowed to be an "algebraic function" then we get the general case. In even just two complex variables this seems not to be known, even with polynomials.

Let us look at the simple $e^{z}=z$. As exponential functions can grow rapidly, one can try to dampen the growth by guessing at $z \approx 2 \pi i k$ for a large integer $k$. With some sort of iterative procedure one gets pretty quickly some sort of expansion

$$
z=2 \pi i k+\log (2 \pi i k)+\frac{\log (2 \pi i k)}{2 \pi i k}-\frac{1}{2} \frac{(\log (2 \pi i k))^{2}}{(2 \pi i k)^{2}}+\frac{\log (2 \pi i k)}{(2 \pi i k)^{2}}+\cdots
$$


So one can well imagine that there infinitely many zeroes, and this can be proved in several ways - see for example Corollary 2.4 (page 794) of Marker [Mar].

Now to two variables. Say we want to solve

$$
e^{z}=w, \quad e^{w}=z
$$

off the diagonal $z=w$. We could eliminate $w$ to get $e^{e^{z}}=z$; but this seems not to be helpful. As above we pick integers $k, h$ and guess $z \approx 2 \pi i k, w \approx 2 \pi i h$; more precisely

$$
z=2 \pi i k+\zeta, \quad w=2 \pi i h+\omega .
$$

We get $e^{\zeta}=2 \pi i h+\omega$ and so a good guess for $\zeta$ is (any determination of) $\log (2 \pi i h)$. Similarly for $\omega$ about $\log (2 \pi i k)$. In fact it turns out now that the resulting approximation

$$
z \approx 2 \pi i k+\log (2 \pi i h), \quad w \approx 2 \pi i h+\log (2 \pi i k)
$$

is good enough for the application of Newton's Method, provided $k, h$ are both large enough and roughly the same size.

Numerically we get after four iterations on $k=100, h=200$ (to avoid the diagonal in (5.2) above) stability up to

$z=7.1374477685 \ldots+629.8842042013 \ldots i, \quad w=6.4456001954 \ldots+1258.1965268827 \ldots i$.

And the method is robust enough that it works for any rational or even algebraic functions on the right-hand sides of $(5.2)$, provided we avoid pairs $(k, h)$ on certain lines; and in any number of variables. Even in just one variable we may solve for example $e^{z}=z+1 / z$ with $k=100$ by $z=6.4455777222788171301981629856188 \ldots+629.87909431073065124541492081115 \ldots i$, or $e^{z}=z+\sqrt{z}$ by $z=6.4738339431980523438931012205386 \ldots+629.85179848455646387241390175467 \ldots i$. Of course the last example is a little suspect with the determination of the square root, so should be written $e^{z}=Z$ with

$$
(Z-z)^{2}=z
$$


In general we make everything precise using algebraic varieties, and then we have the following result.

Proposition 2. Let $\mathcal{X}$ be an affine complex irreducible algebraic variety of dimension $n \geq$ 1. Suppose $f_{1}, \ldots, f_{n}, F_{1}, \ldots, F_{n}$ are rational functions on $\mathcal{X}$ with $f_{1}, \ldots, f_{n}$ algebraically independent and $F_{1}, \ldots, F_{n}$ non-zero. Then there is a point on $\mathcal{X}$ at which the functions are defined with values satisfying

$$
e^{f_{1}}=F_{1}, \ldots, e^{f_{n}}=F_{n} .
$$

The last example above has $\mathcal{X}$ with $n=1$ defined by (5.4) and $f_{1}=z, F_{1}=Z$.

As is well-known, things like Proposition 2 automatically strengthen themselves to give a set of points on $\mathcal{X}$ that is Zariski-dense. For if $G$ is any non-zero rational function on $\mathcal{X}$ then we simply add the equation $e^{g}=G$ on the variety $\mathbf{C} \times \mathcal{X}$ with an extra variable $g$. This ensures that the corresponding value of $G$ is also non-zero.

As might be supposed from the above discussion, our proof actually gives a more structured Zariski-dense set of points. Namely, that the point can be chosen so that $\left(f_{1}, \ldots, f_{n}\right)$ is "relatively near" any one of "sufficiently many" points of $(2 \pi i \mathbf{Z})^{n}$.

Results like Proposition 2 have been mentioned in connexion with Zilber's "Strong Exponential Closure Axiom" for "pseudoexponential fields". Over C this becomes a conjecture, and it is formulated for irreducible algebraic varieties $V$ in $\mathbf{C}^{n} \times\left(\mathbf{C}^{*}\right)^{n}$. A non-strong form, mentioned in Corollary 4.5 (page 83) of [Z2] in connexion with $\mathbf{C}_{\text {exp }}$, states that if $V$ is "normal" (or ex-normal) and "free" then $V$ has a point of the shape $\mathbf{v}=\left(z_{1}, \ldots, z_{n}, e^{z_{1}}, \ldots, e^{z_{n}}\right)$. Our Proposition 2 provides a proof with the sole condition that the projection of $V$ to $\mathbf{C}^{n}$ is dominant. This implies that $V$ is normal (but not that $V$ is free).

The strong form states that for any field $k$ finitely generated over $\mathbf{Q}$ there is such a $\mathbf{v}$ which is a generic point of $V$ over $k$. This is open even for $n=1$, but in that case Marker deduced it from the notorious Schanuel Conjecture when $V$ is defined over the algebraic closure of $\mathbf{Q}$, and more recently Mantova removed the condition on the field of definition. See Theorem 1.6 (page 793) of [Mar] and Theorem 1.2 (page 310) of [Man]. See also [DFT] and $[\mathrm{G}]$.

See also [Z1] section 5 for what is known in $\mathbf{R}^{n} \times\left(\mathbf{R}^{*}\right)^{n}$. 
The following result sums up the iterative procedure needed to prove Proposition 2. Here $\mathbf{p}=\left(p_{1}, \ldots, p_{n}\right)$ are coordinates, and norms of vectors and matrices are (euclidean) $L^{2}$.

Lemma 1. Let $\mathbf{p}_{0}$ be in $\mathbf{C}^{n}$ and let $\Phi=\left(\phi_{1}, \ldots, \phi_{n}\right)$ be an analytic map from some neighbourhood of $\mathbf{p}_{0}$ to $\mathbf{C}^{n}$ whose Jacobian matrix $J(\mathbf{p})$ is non-singular at $\mathbf{p}_{0}$. Define $\mathbf{q}_{0}$ in $\mathbf{C}^{n}$ by

$$
\mathbf{q}_{0} J\left(\mathbf{p}_{0}\right)=-\Phi\left(\mathbf{p}_{0}\right),
$$

and let $U$ be the set of $\mathbf{p}$ with

$$
\left|\mathbf{p}-\mathbf{p}_{0}\right| \leq 2\left|\mathbf{q}_{0}\right| \text {. }
$$

Suppose $\Phi$ is analytic on $U$ satisfying

$$
\sum_{k=1}^{n}\left|H\left(\phi_{k}\right)\right|^{2} \leq M^{2}
$$

for the Hessian matrices and that

$$
2 M\left|\mathbf{q}_{0}\right|\left|J\left(\mathbf{p}_{0}\right)^{-1}\right|<1
$$

for some $M>0$. Then there is $\mathbf{p}$ in $U$ with $\Phi(\mathbf{p})=0$.

Proof. See Theorem 25.1 (page 187) of the second edition [O1] of Ostrowski's book. It is stated for $\mathbf{R}^{n}$, but the proof works also for $\mathbf{C}^{n}$ (this is made even clearer in the third edition [O2] page 274). He uses Newton's Method, paying close attention to constants and for more general $L^{?}$ norms. We take his $\sigma=\rho=2$.

Lemma 2. For $\mathbf{z}=\left(z_{1}, \ldots, z_{n}\right)$ let $\alpha \neq 0$ in the algebraic closure $\overline{\mathbf{C}(\mathbf{z})}$ be an algebraic function. Then there are $q$ in $\mathbf{Z}$, e in $\mathbf{N}$ and a subset $\Gamma$ of $\mathbf{Z}^{n}$, Zariski-dense in $\mathbf{C}^{n}$, with the following property. For each $\gamma$ in $\Gamma$ there is $\epsilon>0$ and $\delta>0$ and functions $c_{p}(\mathbf{w})(p \geq q)$ analytic on the disc $|\mathbf{w}-\gamma|<\epsilon$ with $c_{q}(\gamma) \neq 0$ such that on this disc the Puiseux series $\sum_{p \geq q} c_{p}(\mathbf{w}) t^{-p / e}$ converges absolutely for $|t|>1 / \delta$ to a branch of $\alpha(t \mathbf{w})$.

Proof. In words, the Puiseux expansion along a complex line $\mathbf{z}=t \mathbf{w}$ depends analytically on the line, at least near almost all lines. This surely must be known, but we give a proof sketch.

By Puiseux's Theorem over $\mathcal{K}=\overline{\mathbf{C}(\mathbf{w})}$ there is a formal branch $\alpha(t \mathbf{w})=\sum_{p \geq q} c_{p}(\mathbf{w}) t^{-p / e}$ over $\mathcal{K}$. But it is well-known that the $c_{p}(\mathbf{w})$ lie in a finite extension $K$ of $\mathbf{C}(\mathbf{w})$; for example this follows from a proof given by Cassels [C]. He is actually proving Eisenstein's 
Theorem over $\mathbf{Q}$; but the proof applies much more generally, and shows in our situation that if $\mathcal{O}$ is the integral closure of $R=\mathbf{C}[\mathbf{w}]$ in $K$, then there are non-zero $A, B$ in $R$ such that $A B^{p-q} c_{p}$ lies in $\mathcal{O}$. In the typical case $q \geq 0$ and $e=1$ one only has to assume $f$ in $\mathcal{K}[[T]]$ and $g_{0}, \ldots, g_{J}$ in $\mathbf{C}(\mathbf{w})[T]$ in his Theorem 5.1 (page 28). In the proof $h, h_{1}, \ldots, h_{J}$ end up in $K[T]$ for $K=\mathbf{C}\left(\mathbf{w}, f_{0}, \ldots, f_{m+1}\right)$. The subsequent induction shows that $f_{m+k+1}(k=1,2, \ldots)$ also lie in $K$; but we can even suppose $h, h_{1}, \ldots, h_{J}$ in $\mathcal{O}[T]$, and now the induction shows that $l^{k} f_{m+k+1}(k=1,2, \ldots)$ are in $\mathcal{O}$ with $l=h_{1}(0) \neq 0$ in $\mathcal{O}$. The claim about $A, B$ follows quickly, as well as the extension to arbitrary $q$ and $e$.

It is well-known that $\mathcal{O}=R \omega_{1}+\cdots+R \omega_{d}$ is finitely generated. So all we have to do is represent the $c_{p}$ via $A, B, \omega_{1}, \ldots, \omega_{d}$ as analytic functions near sufficiently many $\gamma$. It suffices to take $A(\gamma) \neq 0$ and $B(\gamma) \neq 0$, and then it really is well-known that we can make $\omega_{1}, \ldots, \omega_{d}$ analytic near almost all $\gamma$. The absolute convergence is routine, and this completes the proof.

Here is a very simple example with $n=2$ and $\alpha=\sqrt{z_{1}+z_{2}}$ (no chance of any Puiseux-type expansion near $\left(z_{1}, z_{2}\right)=(\infty, \infty)$ in this case), with

$$
\alpha\left(w_{1} t, w_{2} t\right)=\sqrt{\left(w_{1}+w_{2}\right) t}=c_{1} t^{1 / 2}
$$

with

$$
c_{1}=c_{1}\left(w_{1}, w_{2}\right)=\sqrt{\gamma_{1}+\gamma_{2}} \sum_{k=0}^{\infty}\left(\begin{array}{c}
1 / 2 \\
k
\end{array}\right)\left(\frac{\left(w_{1}-\gamma_{1}\right)+\left(w_{2}-\gamma_{2}\right)}{\gamma_{1}+\gamma_{2}}\right)^{k}
$$

provided $\gamma_{1}+\gamma_{2} \neq 0$.

We can now prove Proposition 2. But as a warm-up we solve (5.2) with (5.3). Taking new variables

$$
p=z-2 \pi i k-\log (2 \pi i h), \quad q=w-2 \pi i h-\log (2 \pi i k)
$$

we get the equations $\phi(p, q)=\psi(p, q)=0$ with

$$
\phi=e^{p}-\frac{2 \pi i h+\log (2 \pi i k)+q}{2 \pi i h}, \quad \psi=e^{q}-\frac{2 \pi i k+\log (2 \pi i h)+p}{2 \pi i k}
$$

to which we apply Lemma 1 . The Jacobian at $(0,0)$ is

$$
\left(\begin{array}{cc}
1 & -\frac{1}{2 \pi i k} \\
-\frac{1}{2 \pi i h} & 1
\end{array}\right)
$$

near the identity matrix if $k$ and $h$ are large, and

$$
\phi(0,0)=-\frac{\log (2 \pi i k)}{2 \pi i h}, \quad \psi(0,0)=-\frac{\log (2 \pi i h)}{2 \pi i k}
$$


are small if further $k$ and $h$ are about the same size. We could take $k=\gamma m$ and $h=\delta m$ with fixed non-zero integers $\gamma$ and $\delta$, for example, and then the set $U$ has radius of order at most $(\log |m|) /|m|$ for large integers $m$. The Hessians

$$
H(\phi)=\left(\begin{array}{cc}
e^{p} & 0 \\
0 & 0
\end{array}\right), \quad H(\psi)=\left(\begin{array}{cc}
0 & 0 \\
0 & e^{q}
\end{array}\right)
$$

are harmless. Thus for each large integer $m$ we get a solution of (5.2) with errors in (5.3) of order at most $(\log |m|) /|m|$, in harmony with the first three terms of (5.1) for a single variable.

Now to the general proof. Removing a Zariski-closed subset $\mathcal{X}_{0} \neq \mathcal{X}$ from $\mathcal{X}$, we can assume that $\mathcal{X}$ is in some $\mathbf{C}^{N}$ with coordinate functions $x_{1}, \ldots, x_{N}$ and $x_{1}=f_{1}, \ldots, x_{n}=$ $f_{n}$. With $\mathbf{f}=\left(f_{1}, \ldots, f_{n}\right)$ each of $x_{1}, \ldots, x_{N}$ is algebraic over $\mathbf{C}(\mathbf{f})$ on $\mathcal{X}$ so there are algebraic functions $\xi_{j}$ in $\overline{\mathbf{C}(\mathbf{z})}$ with

$$
x_{j}=\xi_{j}(\mathbf{f}) \quad(j=1, \ldots, N)
$$

on $\mathcal{X}$. By Lemma 2 for each $j$ there is a subset $\Gamma_{j}$ of $\mathbf{Z}^{n}$, Zariski-dense in $\mathbf{Z}^{n}$, and branches of $\xi_{j}$ on large complex multiples of each $\mathbf{w}$ near any $\gamma$ in $\Gamma_{j}$. Taking the intersection we can assume all $\Gamma_{j}=\Gamma$. Thus

$$
f_{k}\left(\xi_{1}(\mathbf{z}), \ldots, \xi_{N}(\mathbf{z})\right)=z_{k} \quad(k=1, \ldots, n)
$$

on such multiples. We define $\alpha_{k}=F_{k}\left(\xi_{1}, \ldots, \xi_{N}\right)$ in $\overline{\mathbf{C}(\mathbf{z})}$, so that

$$
F_{k}=\alpha_{k}(\mathbf{f}) \quad(k=1, \ldots, n)
$$

on $\mathcal{X}$; and $\alpha_{k} \neq 0$ because $F_{k} \neq 0$.

By shrinking $\Gamma$ further, we can use Lemma 2 again to get $q_{k}$ in $\mathbf{Z}, e_{k}$ in $\mathbf{N}$ with the following property. For each $\gamma$ in $\Gamma$ there is $\epsilon_{k}>0$ and $\delta_{k}>0$ and functions $c_{p k}(\mathbf{w})\left(p \geq q_{k}\right)$ analytic on the disc $|\mathbf{w}-\gamma|<\epsilon_{k}$ with $c_{q_{k} k}(\gamma) \neq 0$ such that on this disc the Puiseux series $\sum_{p \geq q_{k}} c_{p k}(\mathbf{w}) t^{-p / e_{k}}$ converges absolutely for $|t|>1 / \delta_{k}$ to a branch of $\alpha_{k}(t \mathbf{w})$. We can clearly suppose all $\epsilon_{k}=\epsilon$ and all $\delta_{k}=\delta$.

Pick $\gamma$ in $\Gamma$. Let $m$ be in $\mathbf{Z}$ with $|t|>1 / \delta$ for $t=2 \pi i m$; we will eventually make $m$ even larger. Then

$$
\alpha_{k}(2 \pi i m \gamma)=\sum_{p \geq q_{k}} c_{p k}(\gamma) t^{-p / e_{k}}=c_{q_{k} k}(\gamma) t^{-q_{k} / e_{k}}\left(1+O\left(|m|^{-1 / e_{k}}\right)\right) \neq 0 \quad(k=1, \ldots, n)
$$


if $|m|$ is sufficiently large. Fix some principal value $\lambda_{k}$ of $\log \alpha_{k}(2 \pi i m \gamma)$, so that

$$
\left|\lambda_{k}\right|=O(\log |m|) \quad(k=1, \ldots, n)
$$

and put $\lambda=\left(\lambda_{1}, \ldots, \lambda_{n}\right)$. We would like to apply Lemma 1 to the functions

$$
\phi_{k}(\mathbf{p})=e^{p_{k}}-\frac{\alpha_{k}(2 \pi i m \gamma+\lambda+\mathbf{p})}{\alpha_{k}(2 \pi i m \gamma)} \quad(k=1, \ldots, n)
$$

as in (5.8), but we should check branch compatibility of the numerator. In fact

$$
2 \pi i m \gamma+\lambda+\mathbf{p}=2 \pi i m \mathbf{w}
$$

with

$$
\mathbf{w}=\gamma+\frac{\lambda}{2 \pi i m}+\frac{\mathbf{p}}{2 \pi i m}=\gamma+O\left(\frac{\log |m|}{|m|}\right)
$$

for say $|\mathbf{p}| \leq 1$, so again we are alright if $|m|$ is sufficiently large.

Thus in Lemma 1 we are taking $\mathbf{p}_{0}=0$. We now have to calculate the Jacobian. We note that the numerator in (5.14) is $\sum_{p \geq q_{k}} c_{p k}(\mathbf{w}) t^{-p / e_{k}}$ with $\mathbf{w}$ given by (5.15). The derivative with respect to $p_{r}$ is

$$
\frac{1}{2 \pi i m} \sum_{p \geq q_{k}} \frac{\partial c_{p k}}{\partial p_{r}}(\mathbf{w}) t^{-p / e_{k}} .
$$

It is easy to see that this is $O\left(|m|^{-1-q_{k} / e_{k}}\right)$; for example the $c_{p k}$ grow like at most $C^{p}$ near $\gamma$ and so by Cauchy a similar bound holds for their derivatives. And by (5.13) we see that the denominator in (5.14) is asymptotic to a non-zero multiple of $m^{-q_{k} / e_{k}}$. It follows that $J\left(\mathbf{p}_{0}\right)$ is the identity matrix with error $O\left(|m|^{-1}\right)$, as in (5.9).

A similar argument with Cauchy on the $c_{p q}(\mathbf{w})-c_{p q}(\gamma)$ at $\mathbf{p}=\mathbf{p}_{0}$, also using the asymptotics for the same denominator together with (5.15), shows that $\Phi\left(\mathbf{p}_{0}\right)=O(\log |m| /|m|)$ as in (5.10).

It follows from (5.5) that $\mathbf{q}_{0}=O(\log |m| /|m|)$ in Lemma 1, so again $U$ has radius $O(\log |m| /|m|)$.

And similar arguments to get at the $\frac{\partial^{2} c_{p k}}{\partial p_{r} \partial p_{s}}$ show that the second derivatives of the quotients in (5.14) are small. However taking into account the $e^{p_{k}}$ gives the estimate $M=O(1)$ in Lemma 1 as in (5.11), which suffices for us.

Now the condition (5.7) is satisfied for $|m|$ sufficiently large. This means there is $\mathbf{p}$ with $\Phi(\mathbf{p})=0$ near $\mathbf{p}_{0}=0$; in fact we have (5.6). 
Finally put $\mathbf{z}=2 \pi i m \gamma+\lambda+\mathbf{p}$ and $x=\left(\xi_{1}(\mathbf{z}), \ldots, \xi_{N}(\mathbf{z})\right)$ in $\mathcal{X}$. Since $\gamma_{k}$ is in $\mathbf{Z}$ we see by (5.12) and the definition of $\lambda_{k}$ that

$$
e^{f_{k}(x)}=e^{2 \pi i m \gamma_{k}+\lambda_{k}+p_{k}}=\alpha_{k}(2 \pi i m \gamma) e^{p_{k}}
$$

By the vanishing of (5.14) and the definition of $\alpha_{k}$ this is

$$
\alpha_{k}(2 \pi i m \gamma+\lambda+\mathbf{p})=F_{k}(x)
$$

The proof of Proposition 2 is thereby complete.

6. Proof of Theorem 2. We have to show that $\phi(z)=P_{0}(z)+P_{1}(z) e^{z}$ can have $2 L$ repeated zeroes, not quite the expected $N=L M+L+M=2 L+1$. In fact if $P_{0}, P_{1}$ do not both vanish, then it cannot have $2 L+1$ repeated zeroes, because these would be zeroes of

$$
\left(P_{1}+P_{1}^{\prime}\right) \phi-P_{1} \phi^{\prime}=P_{0} P_{1}+P_{0} P_{1}^{\prime}-P_{0}^{\prime} P_{1}=Q
$$

of degree at most $2 L$, forcing $Q=0$ and so by the easy part of Proposition $1 P_{0}=0$ or $P_{1}=0$, both of which are clearly impossible.

We return to the discussion in section 1 , now with $M=1$ so

$$
\phi=P\left(z, e^{z}\right)=P_{0}(z)+P_{1}(z) e^{z}, \quad \phi^{\prime}=P^{\#}\left(z, e^{z}\right)=P_{0}^{\prime}(z)+\left(P_{1}(z)+P_{1}^{\prime}(z)\right) e^{z} .
$$

There $Q$ was the resultant of

$$
P=P_{0}+P_{1} Y, \quad P^{\#}=P_{0}^{\prime}+\left(P_{1}+P_{1}^{\prime}\right) Y
$$

with respect to $Y$, none other than our $Q$ just above. The strategy is as follows. We have to find $a_{1}, \ldots, a_{2 L}$ and $P_{0}, P_{1}$ with $\phi\left(a_{k}\right)=\phi^{\prime}\left(a_{k}\right)=0(k=1, \ldots, 2 L)$. These imply $Q\left(a_{k}\right)=$ $0(k=1, \ldots, 2 L)$. In fact we obtain this consequence first, by starting with $a_{1}, \ldots, a_{2 L}$ and using the hard part of Proposition 1 to find $P_{0}, P_{1}$ with $Q=\left(X-a_{1}\right) \cdots\left(X-a_{2 L}\right)$. The coefficients of $P_{0}, P_{1}$ are (complicated) algebraic functions of $a_{1}, \ldots, a_{2 L}$. So now the exponential-algebraic equations $\phi\left(a_{k}\right)=0(k=1, \ldots, 2 L)$ can be solved for $a_{1}, \ldots, a_{2 L}$ by our Proposition 2. By Zariski-denseness we can secure $P_{1}\left(a_{k}\right) \neq 0(k=1, \ldots, 2 L)$ and by (6.1) we conclude $\phi^{\prime}\left(a_{k}\right)=0(k=1, \ldots, 2 L)$ as well.

To make this more precise we write $\kappa$ for the map from $\mathbf{C}^{L} \times \mathbf{C}^{L}$ to $\mathbf{C}^{2 L}$ obtained from Proposition 1 by restricting to monic $P_{0}, P_{1}, Q$ (of degrees $L, L, 2 L$ respectively). 
This stays surjective. We also define a map $\sigma$ from $\mathbf{C}^{2 L}$ to $\mathbf{C}^{2 L}$ that "takes zeroes to coefficients"; that is

$$
\sigma\left(z_{1}, \ldots, z_{2 L}\right)=\left(X-z_{1}\right) \cdots\left(X-z_{2 L}\right) .
$$

We define a variety in $\mathbf{C}^{L} \times \mathbf{C}^{L} \times \mathbf{C}^{2 L}$ by

$$
\kappa\left(P_{0}, P_{1}\right)=\sigma(Z)
$$

for $Z=\left(z_{1}, \ldots, z_{2 L}\right)$. For example with $L=1, P_{0}=p+X, P_{1}=q+X, Z=(a, b)$ we get

$$
p q+p-q=a b, \quad p+q=-a-b ;
$$

easily seen to be irreducible.

Presumably it is irreducible in general, but this may not be straightforward to prove. For if we replace $\kappa\left(P_{0}, P_{1}\right)=P_{0} P_{1}+P_{0} P_{1}^{\prime}-P_{0}^{\prime} P_{1}$ just by $P_{0} P_{1}$, we get

$$
P_{0} P_{1}=\left(X-z_{1}\right) \cdots\left(X-z_{2 L}\right),
$$

where there are exactly $\left(\begin{array}{c}2 L \\ L\end{array}\right)$ components according to the choices of zeroes of say $P_{0}$ (in the above $p=-a, q=-b$ and $p=-b, q=-a)$.

Fortunately it turns out that we can work on any component $\mathcal{X}$ of (6.2). On $\mathcal{X}$ already each $\left(P_{0}, P_{1}\right)$ determines at most finitely many $Z$, so its dimension $n \leq 2 L$. On the other hand the ideal of equations (6.2) in $4 L$ variables has $2 L$ generators, so $n \geq 4 L-2 L=2 L$ (see for example Theorem 30 of [ZS] page 240). Thus $n=2 L$; and the coefficients of $P_{0}, P_{1}$ are algebraically independent on $\mathcal{X}$. It follows from Proposition 1 that also $z_{1}, \ldots, z_{2 L}$ are algebraically independent on $\mathcal{X}$; that is why we described it so in section 4 .

Next we show that the functions $P_{1}\left(z_{k}\right)(k=1, \ldots, 2 L)$ do not vanish on $\mathcal{X}$. This is essentially due to the symmetry in $\sigma$. In fact otherwise by the Nullstellensatz some $P_{1}\left(z_{k}\right)^{e}$ would lie in the ideal of the equations (6.2). But then interchanging $z_{k}, z_{j}$ would show that also $P_{1}\left(z_{j}\right)^{e}$ does. Thus all the $P_{1}\left(z_{j}\right)=0(j=1, \ldots, 2 L)$ on $\mathcal{X}$. However a polynomial of degree $L \geq 1$ cannot have $2 L$ different zeroes.

Similarly for the $P_{0}\left(z_{k}\right)(k=1, \ldots, 2 L)$. And also the resultant $R$ of $P_{0}, P_{1}$ does not vanish on $\mathcal{X}$.

We now apply the Zariski-dense version of Proposition 2 to solve the system

$$
P_{1}\left(z_{k}\right) \neq 0, \quad e^{z_{k}}=-\frac{P_{0}\left(z_{k}\right)}{P_{1}\left(z_{k}\right)} \quad(k=1, \ldots, 2 L)
$$


(of course the other variables $z_{j}$ are hidden in $P_{0}, P_{1}$ ), say with $Z=\left(a_{1}, \ldots, a_{2 L}\right)$. We may even make sure that $R \neq 0$ and that $a_{1}, \ldots, a_{2 L}$ are distinct.

Thus the resulting function $\phi(z)=P_{0}(z)+P_{1}(z) e^{z}$ has zeroes at $a_{1}, \ldots, a_{2 L}$. So of course does $Q=\kappa\left(P_{0}, P_{1}\right)$. It follows from (6.1) and $P_{1}\left(z_{k}\right) \neq 0$ that $\phi^{\prime}$ also has zeroes there; so these zeroes of $\phi$ are repeated. As $R \neq 0$, the polynomials $P_{0}, P_{1}$ have no common factor. Thus $P_{0}(X)+P_{1}(X) Y$ is irreducible. This proves Theorem 2 .

A bit extra can be squeezed out by relaxing the monic conditions. For example, when $L=1$, a function

$$
\phi(z)=p_{00}+p_{10} z+p_{01} e^{z}+p_{11} z e^{z}
$$

can have two repeated zeroes. But we can even specify one of them. Thus if $a \neq 0$ satisfies

$$
1-\left(2+a^{2}\right) e^{a}+e^{2 a}=0
$$

(it exists by Proposition 2 and we can take it as $-4.501457 \ldots+8.424784 \ldots i$ ) then

$$
\phi(0)=\phi^{\prime}(0)=\phi(a)=\phi^{\prime}(a)=0
$$

when

$$
p_{00}=-a+a e^{a}, p_{10}=1-e^{a}+a e^{a}, p_{01}=a-a e^{a}, p_{11}=-1-a+e^{a} .
$$

As we saw, no $\phi \neq 0$ in (6.3) can have three repeated zeroes.

It would be nice to generalize Theorem 2 to general $M$. But just for $L=1, M=2$ the analogue of $Q$ in Proposition 1 has thirteen terms. It has degree at most 4 . So in this case is there $P$, squarefree or even irreducible, such that $P\left(z, e^{z}\right)$ has four repeated zeroes?

It might be interesting also to study the elliptic or abelian analogues.

\section{References}

[BW] A. Baker and G. Wüstholz, Logarithmic forms and diophantine geometry, Cambridge 2007.

[BM] W.D. Brownawell and D.W. Masser, Multiplicity estimates for analytic functions I, J. reine angew. Math. 314 (1980), 200-216.

[C] J.W.S. Cassels, Local fields, Cambridge 1986. 
[DFT] P. D'Aquino, A. Fornasiero and G. Terzo, Generic solutions of equations with iterated exponentials, preprint December 2015.

[F] N.I. Feldman, Approximation of certain transcendental numbers. II: The approximation of certain numbers connected with the Weierstrass $\wp$-function, Izv. Akad. Nauk. SSSR 15, 153-176 (Russian), English transl. Amer. Math. Soc. Transl. 59 (1966), 246-270.

[G] A. Günaydin, Rational solutions of polynomial-exponential equations, Int. J. Number Theory 8 (2012), 1391-1399.

[Man] V. Mantova, Polynomial-exponential equations and Zilber's conjecture (with an appendix by V. Mantova and U. Zannier), Bull. London Math. Soc. 48 (2016), 309-320.

[Mar] D. Marker, A remark on Zilber's pseudoexponentiation, J. Symbolic Logic 71 (2006), 791-798.

[Mas] D. Masser, Rational values of the Riemann zeta function, J. Number Theory 131 (2011), 2037-2046.

[M] D. Mumford, Algebraic geometry I complex projective varieties, Springer 1976.

[O1] A.M. Ostrowski, Solution of equations and systems of equations, Academic Press 1966.

[O2] A.M. Ostrowski, Solutions of equations in euclidean and Banach spaces, Academic Press 1973.

[P] P. Philippon, Lemmes de zéros dans les groupes algébriques commutatifs, Bull. Soc. Math. France 114 (1986), 355-383.

[W] G. Wüstholz, A new approach to Baker's Theorem on linear forms in logarithms I, in Diophantine approximation and transcendence theory, Springer Lecture Notes $\mathbf{1 2 9 0}$ (1987), pp.189-202.

[ZS] O. Zariski and P. Samuel, Commutative algebra I, Springer 1958.

[Z1] B. Zilber, Exponential sums equations and the Schanuel conjecture, J. London Math. Soc. 65 (2002), 27-44.

[Z2] B. Zilber, Pseudo-exponentiation on algebraically closed fields of characteristic zero, 
Annals of Pure and Applied Logic 132 (2005), 67-95.

W.D. Brownawell: Department of Mathematics, The Pennsylvania State University, University Park, PA 16802 (wdb@math.psu.edu).

D.W. Masser: Departement Mathematik und Informatik, Universität Basel, Spiegelgasse 1, 4051 Basel, Switzerland (David.Masser@unibas.ch).

20th April 2016.

Revised 8th October 2016. 


\section{LATEST PREPRINTS}

No. Author: Title

2016-06 ～M. Dambrine, I. Greff, H. Harbrecht, B. Puig

Numerical solution of the homogeneous Neumann boundary value problem on domains with a thin layer of random thickness

2016-07 G. Alberti, G. Crippa, A. L. Mazzucato

Exponential self-similar mixing by incompressible flows

2016-08 M. Bainbridge, P. Habegger, M. Möller

Teichmüller curves in genus three and just likely intersections in $G^{n}{ }_{m} \times G_{a}^{n}$

2016-09 Gabriel A. Dill

Effective approximation and Diophantine applications

2016-10 J. Blanc, S. Zimmermann

Topological simplicity of the Cremona groups

2016-11 I. Hedén, S. Zimmermann

The decomposition group of a line in the plane

2016-12 J. Ballani, D. Kressner, M. Peters

Multilevel tensor approximation of PDEs with random data

2016-13Ｍ. J. Grote, M. Kray, U. Nahum

Adaptive eigenspace method for inverse scattering problems in the frequency domain

2016-14 H. Harbrecht, M. Peters, M. Schmidlin

Uncertainty quantification for PDEs with anisotropic random diffusion

2016-15 F. Da Lio, L. Martinazzi

The nonlocal Liouville-type equation in $R$ and conformal immersions of the disk with boundary singularities

2016-16 A. Hyder

Conformally Euclidean metrics on $R^{n}$ with arbitrary total $Q$-curvature

2016-17 G. Mancini, L. Martinazzi

The Moser-Trudinger inequality and its extremals on a disk via energy estimates

2016-18 R. N. Gantner, M. D. Peters

Higher order quasi-Monte Carlo for Bayesian shape inversion

Preprints are available under https://math.unibas.ch/research/publications 


\section{LATEST PREPRINTS}

No. Author: Title

2016-19 C. Urech

Remarks on the degree growth of birational transformations

2016-20 S. Dahlke, H. Harbrecht, M. Utzinger, M. Weimar

Adaptive wavelet BEM for boundary integral equations: Theory and numerical experiments

2016-21 A. Hyder, S. Iula, L. Martinazzi

Large blow-up sets for the prescribed Q-curvature equation in the Euclidean space

2016-22 P. Habegger

The norm of Gaussian periods

2016-23 P. Habegger

Diophantine approximations on definable sets

2016-24 F. Amoroso, D. Masser

Lower bounds for the height in Galois extensions

2016-25 W. D. Brownawell and D. W. Masser

Zero estimates with moving targets

Preprints are available under https://math.unibas.ch/research/publications 